14. Romieu I, Fabre A, Fournier A, et al. Postmenopausal hormone therapy and asthma onset in the E3N cohort. Thorax 2010;65:292-7.

15. Taylor B, Mannino D, Brown C, et al. Body mass index and asthma severity in the National Asthma Survey. Thorax 2008;63:14-20.
16. Chinn S. Asthma and obesity: where are we now? Thorax 2003;58:1008-10.

17. Behren JV, Lipsett M, Horn-Ross L, et al. Obesity, waist size and prevalence of current asthma in the Californian Teachers Study cohort. Thorax 2009;64:889-93.
18. Beuther DA, Sutherland ER. Overweight, obesity and incident asthma: a meta-analysis of prospective epidemiologic studies. Am J Respir Crit Care Med 2007;175:661-6.

\title{
Improved survival in COPD: reasons for hope
}

\section{Douglas W Mapel}

Not long ago treatment of chronic obstructive pulmonary disease (COPD) was viewed with a sense of nihilism. ${ }^{1}$ Patients were chronically and irreversibly debilitated. Treatments brought only brief palliative relief, and most had serious side effects. Worst of all, many patients were hopelessly addicted to nicotine, and the frustration of watching patient after patient continue to destroy their lungs in spite of their warnings caused many doctors to give up on even diagnosing the disease. Sceptics could point to the relative lack of efficacy of inhaled steroids in COPD. Cynics could question the motives of those who want to help persons who have destroyed their own health.

The last decade has seen the introduction of new treatments for COPD, which in turn have brought a new perspective and sense of energy. ${ }^{1}$ It is not uncommon in medicine that the discovery of new treatments results in a surge of new interest in a disease. Many examples of this phenomenon are notable in recent history: sleep apnoea, pulmonary hypertension and erectile dysfunction are but a few. The release of the long-acting anticholinergic drug tiotropium, the approval of combined long-acting $\beta_{2}$ agonist/corticosteroid inhalers for COPD, development of new nebulised agents and release of innovative medications for nicotine addiction have provided us with new effective tools for COPD. Advocacy groups for COPD have multiplied and grown worldwide. In countries where it is allowed, direct-to-consumer drug advertising has introduced hundreds of millions of lay persons to the term 'COPD', and has educated doctors and patients alike that there are effective new treatments that can

Correspondence to Dr Douglas W Mapel, Lovelace Clinic Foundation, 2309 Renard Place SE, Suite 103, Albuquerque, NM 87106-4264, USA;

dmapel@comcast.net reduce chronic respiratory symptoms and improve lung function.

An important dimension of COPD outcomes research that is lacking is evidence that treatment also improves survival. Survival is a big motivator. Patients are willing to tolerate expensive treatments with horrible side effects if they believe that they will improve their survival (eg, chemotherapy for cancer). Doctors will screen for asymptomatic diseases if they believe that finding them will improve the patient's chances of survival (eg, elevated cholesterol). The US Preventive Services Task Force recommended against using spirometry to screen for COPD in part due to the lack of evidence that pharmacological treatments affect hospitalisations or all-cause mortality. ${ }^{2}$

In this issue of Thorax, Almagro et al (see page 298) provide new evidence that modern management of COPD patients does improve survival. ${ }^{3}$ They compared the long-term survival of patients admitted for a COPD exacerbation in one hospital during the period from October 1996 to May 1997 ( $n=135)$ with that of patients with COPD admitted to the same hospital from June 2003 to September 2004 $(\mathrm{n}=181)$. Unadjusted 3-year mortality decreased from $47.4 \%$ to $38.7 \%(p<0.05)$. The cohorts were very similar in terms of demographics, and while the earlier group had a slightly worse airflow obstruction (forced expiratory volume in $1 \mathrm{~s}\left(\mathrm{FEV}_{1}\right) \%$ predicted $41.4 \%$ vs $45.1 \%$ ), the latter group had worse oxygenation (partial pressure of oxygen $\left(\mathrm{PO}_{2}\right)$ at discharge 70 vs 63$)$ and more severe dyspnoea. After adjustment for multiple clinical factors, the relative risk of death was 0.70 , which did not quite reach the definition of statistical significance (95\% CI 0.47 to 1.05). Nevertheless, in a relatively small population, this is an impressive survival difference. Mortality was significantly decreased among the patients with COPD who also had concomitant heart failure or cancer, suggesting that management of comorbidities may have had as much to do with the improved survival as increased use of long-acting $\beta_{2}$ agonists and tiotropium at hospital discharge.

The Almagro study is obviously limited by the factors that affect any historical study design, including the potential for selection biases and possibility of inadequate adjustment for confounders. Also, the study is not conclusive proof that better cancer and heart failure care or better respiratory drugs are the causes of the improved survival, even though there were substantial changes in their utilisation between these two time periods. On the other hand, the features of a relatively consistent patient population, similar prevalence of co-morbidites and treatment in one hospital system provide some reassurance that the risks for selection and treatment biases were minimised. Despite the shortcomings, the message for the providers and patients in this hospital and similar systems is clear: we can improve survival in $\mathrm{COPD}$, and that is very exciting news.

Smoking cessation for those who are still smoking and oxygen therapy for those who are chronically hypoxaemic are still the only treatments clearly proven to improve survival in COPD. ${ }^{4}$ Only one randomised clinical trial for treatment of COPD, the TORCH study, has ever been designed with survival as the primary end point, and it missed its statistically defined definition of a significant difference by the narrowest of margins (observed $p$ value 0.052). ${ }^{5}$ The UPLIFT study of tiotropium in moderate and severe COPD found a survival benefit, but survival was a secondary end point and thus it has not received much attention. ${ }^{6}$ Also, in UPLIFT the statistical significance was observed at the end of the protocoldefined 4-year treatment period $(p=0.034)$, but not 30 days thereafter $(p=0.086)$, which is a perplexing observation that raises questions about withdrawal from treatment. The INSPIRE study was a direct comparison of salmeterol plus fluticasone in a combined inhaler (SFC) versus the long-acting anticholinergic tiotropium in severe COPD, with exacerbations captured as the primary outcome. ${ }^{7}$ Both treatments had a similar impact on COPD 
exacerbation rate, but SFC users had a statistically significant reduction in allcause mortality. Survival in the INSPIRE study was captured as a safety measure, so this observation has also not received much attention. Survival is a difficult and expensive outcome to capture as a primary outcome in a randomised clinical trial, and unfortunately we are not likely to see more survival studies in COPD any time soon.

Several retrospective studies have examined the survival benefit of specific treatments in COPD, with mixed results. Some have found a survival benefit with inhaled corticosteroids with or without long-acting $\beta$ agonists, but others employing very different methods have not found a benefit. ${ }^{89}$ Although these studies provide insight into the 'real-life' impact of treatments in the general population, the biases inherent to retrospective study designs and controversies around appropriate analysis techniques leave much uncertain.

It is interesting to speculate on what specific interventions most improve the chances for improved survival in COPD. Is it long-acting maintenance medications? Exercise rehabilitation? Improved attention to co-morbidities? Perhaps we will never prove that any single invention is sufficient by itself; maybe it takes multiple approaches implemented simultaneously. Whatever the case, success begins with belief by the doctor that treatment is effective, and confidence that we can make a difference for our patients with COPD.

The only cheap and universal cure. - Abraham Cowley

Competing interests DWM is the recipient of research grants from GlaxoSmithKline, Pfizer and AstraZeneca.

Provenance and peer review Commissioned; not externally peer reviewed.

Thorax 2010;65:284-285.

\section{REFERENCES}

1. Celli BR. Update on the management of COPD. Chest 2008;133:1451-62.

2. US Preventive Services Task Force. Screening for chronic obstructive pulmonary disease using spirometry: U.S. Preventive Services Task Force
Hope! Of all ills that men endure,

doi:10.1136/thx.2009.128330

recommendation statement. Ann Intern Med 2008;148:529-34.

3. Almagro P, Salvadó M, Garcia-Vidal C, et al. Recent improvement in long-term survival after a COPD hospitalisation 2010;65:298-302.

4. Global Initiative for Chronic Obstructive Lung Disease (GOLD). Global Strategy for Diagnosis, Management and Prevention of COPD. Updated 2009. www.goldcopd.com (accessed 4 Dec 2009).

5. Calverley PM, Anderson JA, Celli B, et al. Salmeterol and fluticasone propionate and survival in chronic obstructive pulmonary disease. N Engl J Med 2007;356:775-89

6. Celli B, Decramer M, Kesten S, et al; UPLIFT Study Investigators. Mortality in the 4-year trial of tiotropium (UPLIFT) in patients with chronic obstructive pulmonary disease. Am J Respir Crit Care Med 2009;180:948-55.

7. Wedzicha JA, Calverley PM, Seemungal TA, et al; INSPIRE Investigators. The prevention of chronic obstructive pulmonary disease exacerbations by salmeterol/fluticasone propionate or tiotropium bromide. Am J Respir Crit Care Med 2008;177:19-26.

8. Sin DD, Wu L, Anderson JA, et al. Inhaled corticosteroids and mortality in chronic obstructive pulmonary disease. Thorax 2005;60:992-7.

9. Mapel DW, Nelson LS, Lydick E, et al. Survival among COPD patients using fluticasone/salmeterol in combination versus other inhaled steroids and bronchodilators alone. COPD 2007; 4:127-34

\section{Lung alert}

\section{Selective proteasome inhibitors may provide a novel therapeutic approach to Mycobacterium tuberculosis}

Proteasomes are large protein complexes that are structurally conserved across eukaryote cells including mycobacteria. They are needed to degrade unneeded or damaged proteins and, as such, have a vital role in cellular homeostasis. The cytotoxicity of proteasome inhibitors has been demonstrated by bortezomib (Velcade), which was the first therapeutic proteasome inhibitor and is licensed for use against multiple myeloma. Unfortunately, non-selective proteasome inhibitors are inherently toxic, limiting their potential use. In the present study the authors screened over 20000 compounds to identify two oxathiazol-2-one inhibitors-GL5 and HT117-that were able to cross the cell wall of Mycobacterium tuberculosis and inhibit proteasome activity. These compounds were compared with bortezomib for efficacy and toxicity. Measured against the BCG strain, at equal concentrations GL5 and HT117 inhibited around $90 \%$ of mycobacterial proteasome activity compared with $52 \%$ achieved by bortezomib. Both compounds dose-dependently killed $M$ tuberculosis over 4 days in synergy with nitric oxide (which at sub-lethal levels renders $M$ tuberculosis non-replicative). Furthermore, GL5 and HT117 were non-toxic to mammalian cells when given at concentrations 3000-fold greater than those at which bortezomib killed monkey epithelial cells. In kinetic studies, oxathiazol-2-one compounds inhibited $M$ tuberculosis proteasomes irreversibly.

Non-replicating $M$ tuberculosis displays relative resistance to conventional antibiotics. Mycobactericidal, non-cytotoxic proteasome inhibitors that act irreversibly may provide a novel therapeutic tool against $M$ tuberculosis as well as a synergistic adjunct to conventional anti-tuberculosis medications.

- Lin G, Li D, Sorio de Carvalho LP, et al. Inhibitors selective for mycobacterial versus human proteasomes. Nature 2009;:461:621-6.

\section{S Mahendran}

Correspondence to S Mahendran, SpR Respiratory Medicine, East Sussex Hospitals, UK; drsivamahendran@gmail.com Thorax 2010;65:285. doi:10.1136/thorax.2010.134817 